\title{
Ordered structures with point-like defects of various shapes in magnetic fluid films
}

\author{
Chin-Yih Hong ${ }^{\mathrm{a}}$, Y.S. Yeh ${ }^{\mathrm{a}}$, S.Y. Yang ${ }^{\mathrm{b}}$, H.E. Horng ${ }^{\mathrm{b}, *, 1}$, H.C. Yang \\ a Department of Mechanical and Automation Engineering, Da-Yeh University, Chang-Hwa 515, Taiwan, ROC \\ ${ }^{\mathrm{b}}$ Institute of Electro-Optical Science and Technology, National Taiwan Normal University, Taipei 116, Taiwan, ROC \\ ${ }^{\mathrm{c}}$ Department of Physics, National Taiwan University, Taipei 106, Taiwan, ROC
}

Received 29 August 2003; received in revised form 23 April 2004

Available online 1 June 2004

\begin{abstract}
Owing to the increasing importance of the photonic crystals in opto-electronics, the fabrication of ordered structures becomes urgently required. In this work, we report the formation of the two-dimensional hexagonal ordered structure in the highly homogeneous magnetic fluid film when a magnetic field is applied perpendicularly to the film surface. Furthermore, we investigate the structural patterns when point-like defects of various shapes are introduced artificially into the film. It was found that, for the triangle or the hexagonal defects, a nearly perfect ordered structure is obtained around the defects in the magnetic fluid film, whereas twin boundaries exist for rectangular defects. The existence of the twin boundaries is attributed to the mismatch in the characterized angles of the rectangular defect $\left(=90^{\circ}\right)$ and the hexagonal ordered structure $\left(=60^{\circ}\right)$ in the magnetic fluid film. These observed results reveal the promising ordered structures for developing photonic-crystal cavities by using the magnetic fluid films with point-like cavities.
\end{abstract}

(C) 2004 Elsevier B.V. All rights reserved.

PACS: 75.50.Mm; 75.10.-b; 75.30.-m

Keywords: Magnetic fluid; Ordered structure; Defects

\section{Introduction}

Owing to the versatile applications in optical engineering, the ordered structure is a very

\footnotetext{
*Corresponding author. National Taiwan Normal University, Institute of Electro-optical Science and Technology, 88, Sec. 4, Ting-Chou Road, Taipei 116, Taiwan. Tel.: + 886-229338260; fax: + 886-286-631954.

E-mail address: phyfv001@scc.ntnu.edu.tw (H.E. Horng).

${ }^{1}$ Also with Department of Physics, National Taiwan Normal University, Taipei 116, Taiwan, ROC.
}

important issue in academic and industrial areas of research and development. The ordered structure can be fabricated artificially via laser exposure, photolithography technologies or naturally formed through self-assembling processes [1-3]. Because of the diffraction and interference effect, the ordered structures can be utilized as gratings $[4,5]$, filters [6] or other novel optical devices [7,8]. Recently, with the particular advantage of the ability to localize and control the light flow [9], the photonic crystals become significant in 
electro-optics and attract a lot of interest of researchers. Intuitively, the photonic crystal is constructed with an ordered structure, in which the dielectric or the magnetic permeability functions vary periodically. The period of the permeability function may range from several micrometers to sub-micrometers. Thus, the microwave or the infrared light can propagate in the photonic crystal like the conduction of electrons in crystals $[10,11]$.

In general, the present photonic crystal cavities are made of semiconductors [10-14]. Although many excellent works have been reported on the semiconductor photonic-crystal micro-cavity [1214], the complicated fabrication processes and the lack of tunability make it difficult for wide applications based on semiconductor photonic crystal cavities. Thus, the exploration of a tunable ordered structure is urgent for photonic crystal cavities. Although several directions to achieve tunability of the solid photonic crystals have been explored, most of these ways result in few percent variation of the lattice constant [15-17]. To enhance the tunability of photnic crystals, a highly flexible ordered structure is required. This stirs the interest in colloidal particles because of the expectation that they may provide high tunability by external fields.

Recently, Saado et al. reported stable ordered arrays of millimeter-size magnetic-particle clusters floating on a liquid surface [18]. This ordered structure exhibits photonic stopband at microwave frequencies [19]. The stopband can be continuously tuned until complete disappearance via adjusting the period of the ordered structure by external magnetic field. However, from the application point of view, these photonic crystals are too big to be useful, and also the period of the ordered arrays is hardly reduced for the application in infrared and optical range. According to our previous work [5,20,21], a self-assembling tunable ordered structure can be formed in a highly homogeneous magnetic fluid film. With the demand of the minimization of energy of this system, the ordered structure exhibits a hexagonal array. Instead of millimeter range reported by Saado et al., the period of the ordered structure in the magnetic fluid film ranges from several micrometers to sub-micrometers. This implies that this self-assembling ordered structure may be useful for infrared and optical applications.

One of the innovative applications based on photonic crystals is to introduce a point defect into the ordered structure. The photonic crystal with a point defect acts as a resonant cavity and is promising to enhance the light extraction of solid light emission diodes or laser diodes [12-14]. As a result, the photonic-crystal cavities have been widely developing for the applications in telecommunication, laser engineering, optical integrated circuit, and so on. But, it is well known that it is very difficult to implant defects into self-assembling photonic crystals for fabricating devices. In this work, we develop a fabrication technique to achieve the architecture of a photonic crystal cavity via artificially introducing a point-like hexagonal defect into the magnetic fluid film, and then investigate the tunability of the structural patterns around the defect. Furthermore, the hexagonal defect is replaced with various geometric shapes of defects and the structures around them are examined.

\section{Experimental details}

The magnetic fluid used was kerosene-based ferrofluid possessing magnetic nano-particles of $\mathrm{MnFe}_{2} \mathrm{O}_{4}$, and was prepared with the chemical coprecipitation method. The average value of the particles diameters is $12.6 \mathrm{~nm}$ with a standard deviation of $2.5 \mathrm{~nm}$. To form a magnetic fluid film, the ferrofluid was injected into a hexagonal cell and then covered with a glass plate. The magnetic fluid can be well sealed inside the cell without smearing the cell surface. In this work, the cell was made of silicon wafer. Through a standard photolithography process with the aid of ICPRIE, hexagonal micro-cells of several micrometers in depth were obtained on the silicon wafer. To achieve the architecture of the micro-cavity with a point-like defect at the center of the film, a rod of silicon, which acted as the defect, was fabricated at the center of a cell through the photolithography process. A three-dimensional image and the top view of the micro-cavity with a hexagonal 
point-like defect are shown in Figs. 1(a) and (b). The cross section following the dashed line labeled in Fig. 1(b) is also shown in Fig. 1(c). The result reveals a value of $1.3 \mu \mathrm{m}$ for the depth of the cell. The pictures in Fig. 1 were taken by using a highresolution interference microscope (Zygo 5020). Remarkably, the required shape and size of the silicon rod can be obtained during the photolithography process. The magnetic fluid thin-film micro-cavities were then put into a pair of solenoids, which provided magnetic fields perpendicular to the plane of the films. The variation of the field strength over the cell is less than $0.4 \%$. An optical microscope and a CCD camera were used

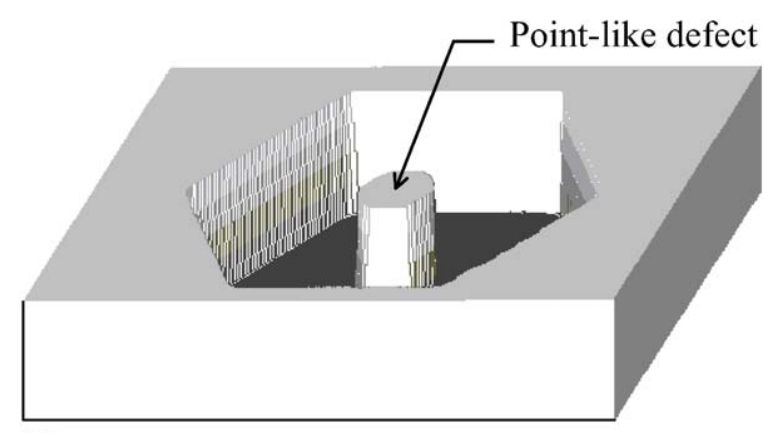

(a)

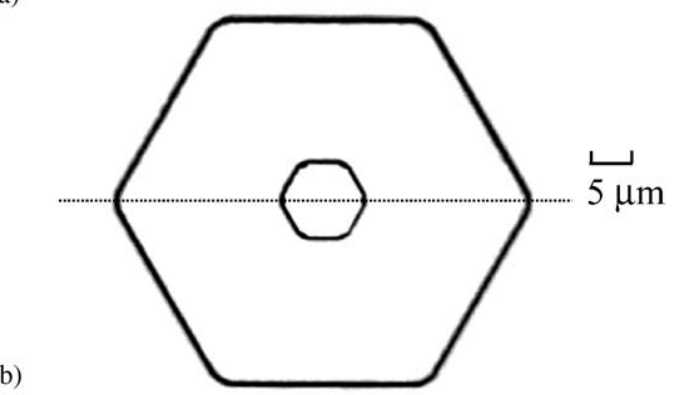

(b)

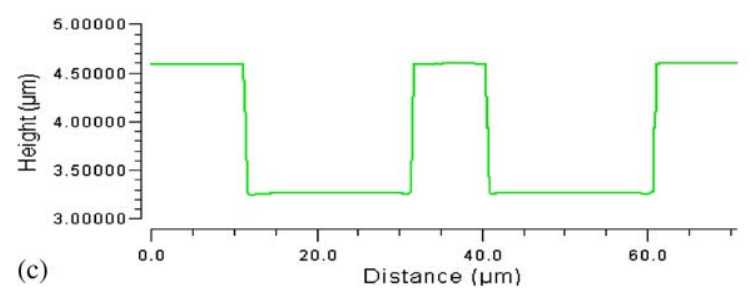

Fig. 1. (a) Three-dimensional, (b) top view and (c) cross section of the cell for the magnetic fluid thin-film micro-cavity. The point-like defect here is hexagonal. The cell is made of silicon wafer via a standard photolithography procedure. The cross section in (c) is guided by the dashed line labeled in (b). to record the structure in the magnetic fluid microcavities.

\section{Results and discussion}

To investigate the structures in the magnetic fluid films with point-like defects, an external magnetic field $H$ perpendicular to the film surface was applied. As the $H$ was increased at a given sweep rate, say $10 \mathrm{Oe} / \mathrm{s}$, to exceed a critical strength ( $\sim 30$ Oe for the investigated sample), magnetic particles $\mathrm{MnFe}_{2} \mathrm{O}_{4}$, which were originally mono-dispersed in the fluid, started to agglomerate to form magnetic columns in the film. The columns were observed to extend from the top to bottom surfaces of the cell. Under higher $H$ 's, more columns appeared and the interaction among magnetic columns was enhanced. Finally when the filed strength reaches to $60 \mathrm{Oe}$, a twodimensional hexagonal ordered structure was achieved in the film, as shown in Fig. 2(a) for the film with a hexagonal point-like defect. The black dots in Fig. 2(a) denote the cross section of the columns. Each vortex of the hexagonal structure is occupied by a magnetic column. This ordered structure shown in Fig. 2(a) displays the architecture of the photonic-crystal micro-cavity.

When the $H$ was further raised, new columns were formed and the columns became closer, as shown in Fig. 2(b). This phenomenon reveals the tunability of the ordered structure in the magnetic fluid thin-film cavity by adjusting the field strength. As the $H$ reaches to $96 \mathrm{Oe}$, each column of the ordered structure started to spilt into two columns and the cross-sectional area of a column became smaller. This splitting process was referred as a transition state and kept proceeding when the $H$ increased up to $118 \mathrm{Oe}$. The mechanism related to the column split in the magnetic fluid films has been suggested to the formation of magnetic subdomains inside a magnetic column under a higher field. The detailed discussion on the column splitting is given in Ref. [7]. It is noted that the structural pattern around the defect is still ordered even at the transition state. This can be evidenced with the image of the ordered structure in the magnetic fluid thin-film micro-cavity at the 

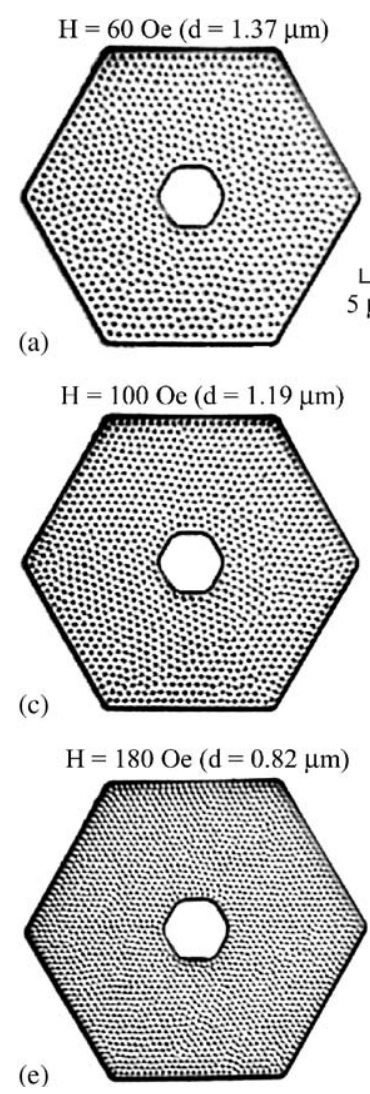

(d)

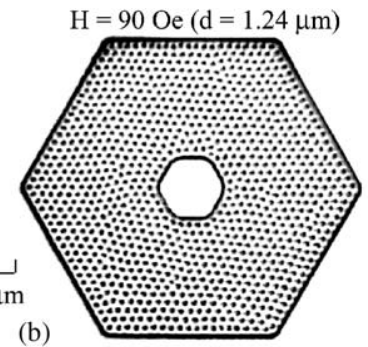

(b)
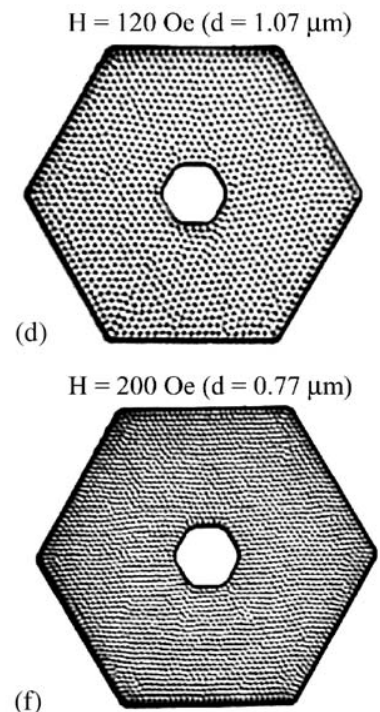

(f)

Fig. 2. (a-f) Structural patterns in the magnetic fluid thin-film micro-cavity under various magnetic field strengths. The magnetic field is outward the sheet. The film thickness $L$ is $1.3 \mu \mathrm{m}$, the concentration of the magnetic fluid is $15.5 \mathrm{emu} / \mathrm{g}$, and the sweep rate of applying field is $10 \mathrm{Oe} / \mathrm{s}$.

transition state shown in Fig. 2(c) for 100 Oe. For $H$ 's over $118 \mathrm{Oe}$, the columns stopped splitting and the more columns were formed with the increasing field strength. As $H$ approached to $130 \mathrm{Oe}$, another transition state occurred. The ordered structural patterns in the magnetic fluid thin-film cavity under 120,180 and 200 Oe are shown in Figs. 2(d)-(f), respectively.

To characterize quantitatively the tunable ordered structure in the magnetic fluid thin-film micro-cavity, the averaged spacing $d$ between two neighboring columns and the averaged diameter $a$ of the cross-sectional area of columns are analyzed. According to the theoretical analysis [17], only the ordered structure surrounding the defect dominates the photonic properties of the photonic-crystal micro-cavity. Thus, the first fourlayered columns surrounding the defect are counted for the values of $d$ and $a$. The images in Fig. 2 reveal definitely a reduction in the column spacing with the increasing $H$, and the corresponding $d-H$ curve for $\mathrm{d} H / \mathrm{d} t=10 \mathrm{Oe} / \mathrm{s}$ is shown in Fig. 3(a). It is clear that two kinks (as indicated by arrows) appear in the $d-H$ curve. These two kinks were found to correspond to the occurrence of the column splitting. The transition states, at which columns split, are also pointed out in Fig. 3. It is worth noting that the decrease in $d$ with varying $H$ outside the transition state is due to the formation of new columns, while the decrease in $d$ at the transition state is attributed to the column

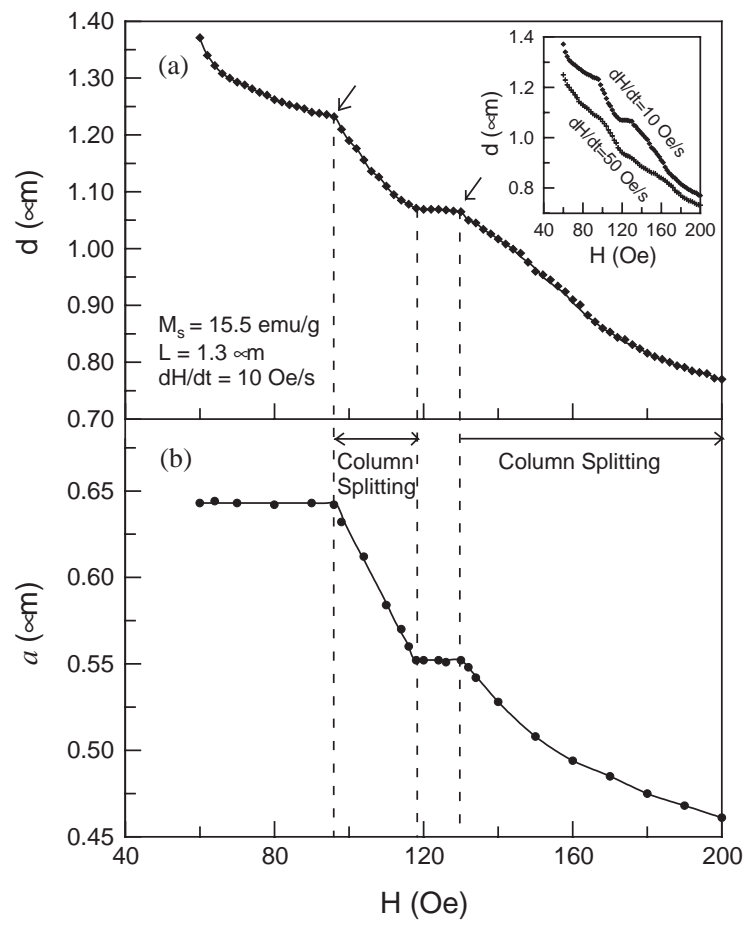

Fig. 3. Averaged (a) column spacing and (b) column diameter of the ordered structure in the magnetic fluid thin-film microcavity versus externally magnetic field strength. The film thickness $L$ is $1.3 \mu \mathrm{m}$, the concentration of the magnetic fluid is $15.5 \mathrm{emu} / \mathrm{g}$, and the sweep rate of applying field is $10 \mathrm{Oe} / \mathrm{s}$. The two kinks indicated by arrows correspond to the occurrence of the splitting state during the structural evolution in the magnetic fluid film under external magnetic fields. The inset in (a) shows the $d-H$ curves of the ordered structure for $\mathrm{d} H / \mathrm{d} t=10$ and $50 \mathrm{Oe} / \mathrm{s}$. 
splitting. On the other hand, at the column splitting state, the column diameter was continuously reduced with the rising field strength, as shown in Fig. 3(b), whereas the column diameter kept unchanged outside the transition states under varying fields. By experimental observation, the shape of magnetic columns is almost round when the field strength is beyond the minimum value necessary for column formation. The results in Fig. 3(a) show that the value of $d$ can be manipulated from 1.37 to $0.77 \mu \mathrm{m}$ as the $H$ increases from 60 to 200 Oe with a sweep rate of $10 \mathrm{Oe} / \mathrm{s}$. The whole $d-H$ curve was observed to be shifted to the region with lower values of $d$ when the sweep rate $\mathrm{d} H / \mathrm{d} t$ is raised, as shown in the inset for $\mathrm{d} H / \mathrm{d} t=10$ and $50 \mathrm{Oe} / \mathrm{s}$. It is believed that the $d$ can be further reduced under higher $H$ 's or $\mathrm{d} H / \mathrm{d} t$ 's. According to the theoretical prediction [6], the wavelength of a resonant light in a photonic crystal cavity is roughly around halfperiod of the ordered structure. Thus, the magnetic fluid thin-film micro-cavity could be able to resonate IR, near IR and visible lights because of tunable $d$ 's.

In addition to the hexagonal point-like defect, various shapes of defects were also fabricated in the magnetic fluid films and the structures were then examined. The shapes of the point-like defects investigated here are hexagonal, triangular, rectangular and circular. It was found that a nearly perfectly single-crystalline structure can be achieved around the hexagonal and the triangular defects in the magnetic fluid thin-film micro-cavity shown in Figs. 4(a1) and (a2), respectively, a multidomain crystalline structure is resulted for the rectangular and the ordered structure deformed around the circular defects, as shown in Fig. 4(a3) and (a4).

To realize the formation of the single- or the multi-crystalline ordered structures of the magnetic fluid films around the various defects, the structural patterns surrounding these defects were drawn and analyzed, as shown in Figs. 4(b)'s. For the hexagonal and the triangular defects in Figs. 4(b1) and (b2), a single domain of the hexagonal structure in the magnetic fluid film is obtained. Thus, a single-crystalline ordered structure can be naturally formed around the defects. Besides, it

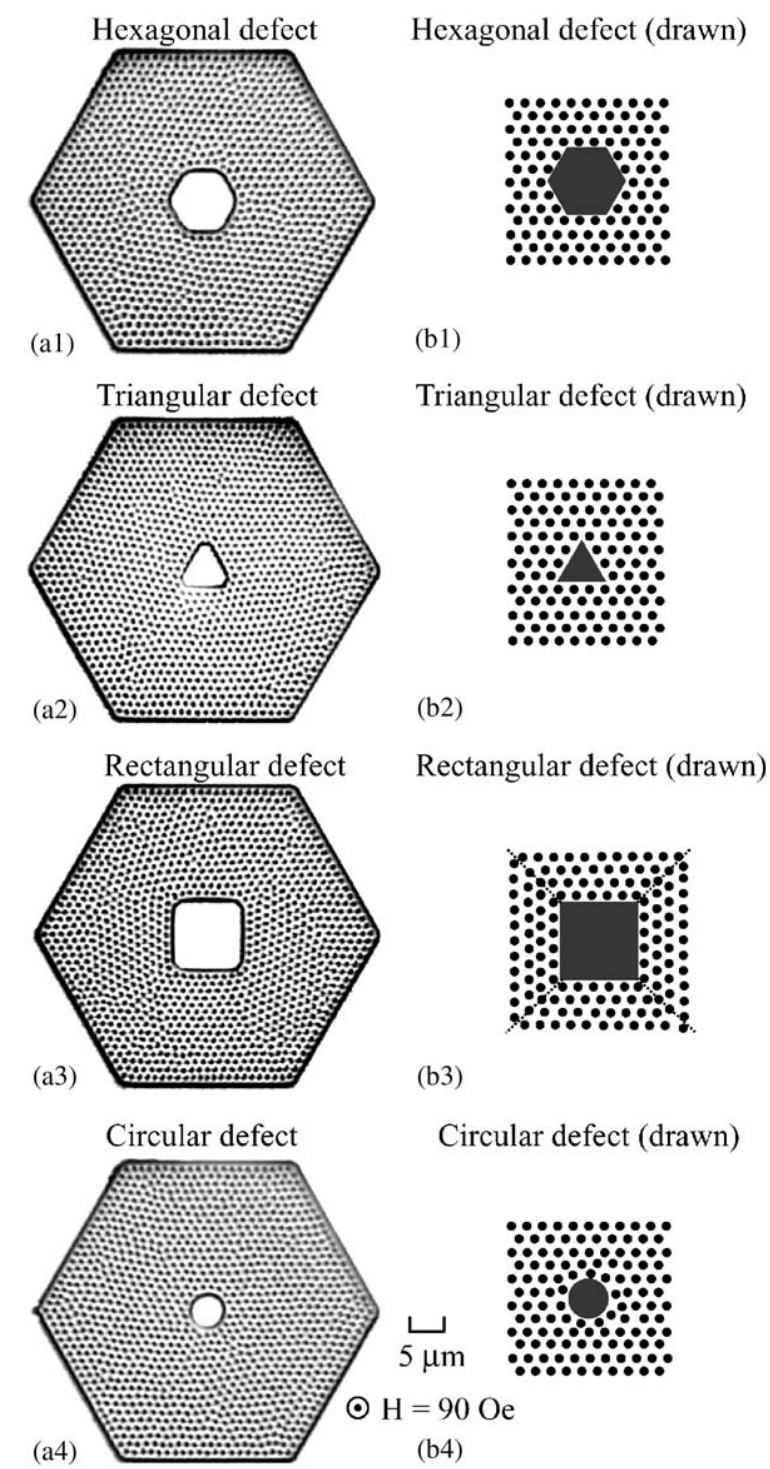

Fig. 4. (a)'s Observed and (b)'s drawn ordered structures in the magnetic fluid thin-film micro-cavities with the hexagonal, triangular, rectangular, and circular point-like defects under 90 Oe. The dashed lines in (b3) denote the twin boundaries. The magnetic field is outward the sheet. The film thickness $L$ is $1.3 \mu \mathrm{m}$, the concentration of the magnetic fluid is $15.5 \mathrm{emu} / \mathrm{g}$, and the sweep rate of applying field is $10 \mathrm{Oe} / \mathrm{s}$.

can be clearly observed that there must exist twins for the crystalline structures surrounding the rectangular defect from the drawn pattern shown in Fig. 4(b3). The twin boundaries result in the multi-crystal lines, as indicated by dashed lines 
Fig. 4(b3). Fig. 4(b4) shows obviously that the ordered structure became deformed when a circular point-like defect existed. Although different types of crystalline structures are obtained in the magnetic fluid thin-film micro-cavities with various shapes of defects, all these ordered structures around these defects can be tunable by well controlling the field strength and exhibit a similar trend for the $d-H$ curves shown in Fig. 3.

\section{Conclusions}

We have successfully fabricated the ordered structures around point-like defects of various shapes by using the highly homogeneous magnetic fluid films. This gives the opportunity to develop the photonic-crystal micro-cavities by utilizing these ordered structures. Furthermore, the period of the ordered structure can be manipulated from micrometers to sub-micrometers by adjusting the externally magnetic field strength or the sweep rate. Therefore, the highly homogeneous magnetic fluid under external magnetic fields affords a chance to design tunable photonic-crystal microcavities.

\section{Acknowledgements}

This work is supported by the National Science Council of ROC under Grant Nos. NSC 92-2112E-212-011 and NSC 92-2112-M-003-010 and also supported with the MOE Program for Promoting Academic Excellent of University under Grant No. 91-N-FA01-2-4-2.

\section{References}

[1] K.O. Hill, G. Meltz, J. Lightwave Technol. 15 (1997) 1263.

[2] M. Locar, T. Doll, J. Vuckoic, A. Scherer, J. Lightwave Technol. 18 (2000) 1402.

[3] S.M. Yang, G.A. Ozin, Chem. Commun. 24 (2000) 2507.

[4] O. Duhem, J.-F. Henninot, M. Warenfhem, M. Douay, Appl. Opt. 37 (1998) 7223.

[5] H.-E. Horng, S.Y. Yang, S.L. Lee, C.-Y. Hong, H.C. Yang, Appl. Phys. Lett. 79 (2001) 350.

[6] T.C. Chen, J.I. Kuo, W.L. Lee, C.C. Lee, Jpn. J. Appl. Phys. 40 (2001) 1.

[7] C.-Y. Rex Hong, H.-E. Horng, H.-C. Yang, W.B. Yeung, US Patent 5,948,321 (1997).

[8] J.-W. Seo, S.J. Park, K.O. Jang, J. Appl. Phys. 85 (1999) 5956.

[9] J.D. Joannopoulos, R.D. Meade, J.N. Winn, Photonic Crystals, Princeton University Press, NJ, 1995.

[10] E. Yablonovitch, J. Opt. Soc. Am. B10 (1993) 283.

[11] S.Y. Lin, J.G. Fleming, D.L. Hetherington, B.K. Smith, R. Biswas, K.M. Ho, M.M. Sigalas, W. Zubrycki, S.R. Kurtz, J. Bur, Nature 394 (1998) 251.

[12] O. Painter, R.K. Lee, A. Scherer, A. Yariv, J.D. O'Brien, P.D. Dapkus, I. Kim, Science 284 (1999) 1819.

[13] T. Yoshie, A. Scherer, H. Chen, D. Huffaker, D. Deppe, Appl. Phys. Lett. 79 (2001) 114.

[14] J. Sabarinathan, P. Bhattacharya, P.-C. Yu, S. Krishna, J. Cheng, D.G. Steel, Appl. Phys. Lett. 81 (2002) 3876.

[15] P. Halevi, F. Ramos-Mendieta, Phys. Rev. Lett. 85 (2000) 1875.

[16] D. Kang, J.E. Maclennan, N.A. Clark, A.A. Zakhidov, R.H. Baughman, Phys. Rev. Lett. 86 (2001) 4052.

[17] A. de Lustrac, F. Gadot, S. Cabaret, J.M. Lourtioz, T. Brillat, A. Priou, E. Akmansoy, Appl. Phys. Lett. 75 (1999) 1625.

[18] M. Golosovsky, Y. Saado, D. Davidov, Appl. Phys. Lett. 75 (1999) 4168.

[19] Y. Saado, M. Golosovsky, D. Davidov, A. Frenkel, Phys. Rev. B 66 (2002) 195108.

[20] C.-Y. Hong, H.-E. Horng, F.C. Kuo, S.Y. Yang, H.C. Yang, J.M. Wu, Appl. Phys. Lett. 75 (1999) 2196.

[21] S.Y. Yang, Y.H. Ke, W.S. Tse, H.E. Horng, C.-Y. Hong, H.C. Yang, J. Magn. Magn. 252 (2002) 290. 